\begin{tabular}{|l|l|}
\hline JN $2 \% 1995(21$ & Page 1 of 1 \\
\hline
\end{tabular}

\begin{tabular}{|l|l|}
\hline $\begin{array}{l}\text { 2. To: (Receiving Organization) } \\
\text { Distribution }\end{array}$ & $\begin{array}{l}\text { 3. From: coriginating Organization) } \\
\text { Criticality and Shielding }\end{array}$ \\
\hline 5. Proj./Prog./Dept./Div.: & $\begin{array}{l}\text { 6. Cog. Engr.: } \\
\text { F. Schmittroth }\end{array}$ \\
\hline
\end{tabular}

8. Originator Remarks:

This document is being distributed for final approval and public release.
4. Related EDT No.:
616560
7. Purchase Order No.:
NA

9. Equip./Component No.:

10. System/Bldg./Fafil ity:

11. Receiver Renarks:

12. Major Assm. Owg. No.:

NA

13. Permit/Permit Application No.: NA

14. Required Response Date:

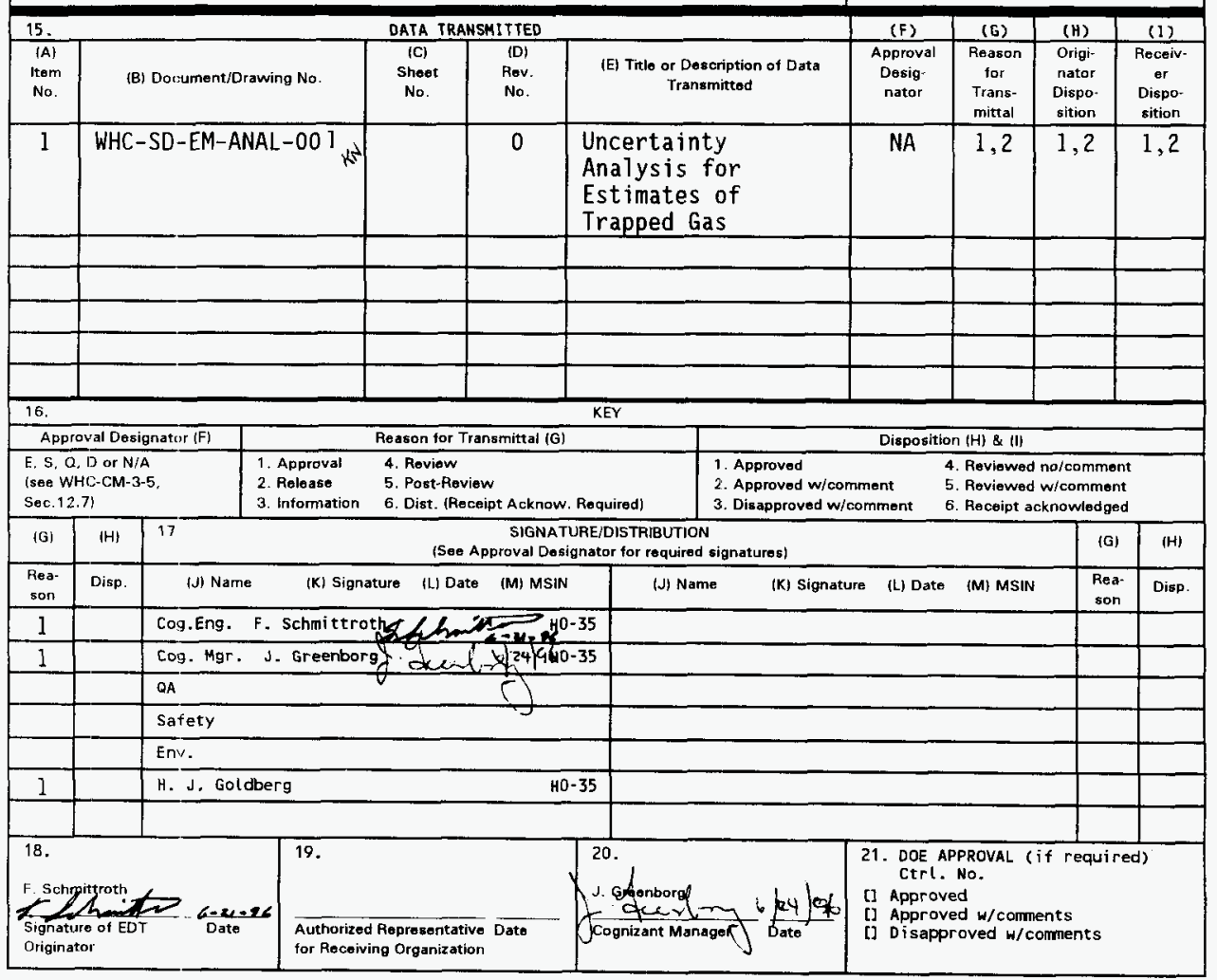

BD-7400-172-2(04/94) GEF097 


\section{Uncertainty Analysis for Estimates of Trapped Gas}

\section{F. Schmittroth}

Westinghouse Hanford Company. Richland. WA 99352

U.S. Department of Energy Contract DE-AC06-87RL10930

$\begin{array}{lll}\text { EDT/ECN: } & 616560 & \text { UC: } 510 \\ \text { Org Code: } & 8 M 730 & \text { Charge Code: } \\ \text { B\&R Code: } & \text { EW3135040 } & \text { Tota1 Pages: } 12\end{array}$

Key Words: Tank Waste, trapped gas, uncertainty

Abstract: An uncertainty anaiys is was made for the amount of trapped gas based on the barometric pressure method for the situation where the method indicates sma11 amounts of gas.

TRADEMARK DISCLAIMER. Reference herein to eny specific commercial product, process, or service by trade nene, trademark, menufacturer, or otherwise, does not necessarily constitute or imply its endorsement, recommendation, or favoring by the United States Goverment or any agency thereof or its contractors or subcontractors.

Printed in the United states of America. To obtain copies of this document, contact: HHC/BCS Document Control Services, P.O. Box 1970, Mailstop H6-08, Richland WA 99352, Phone (509) 372-2420; Fex (509) 376-4989.
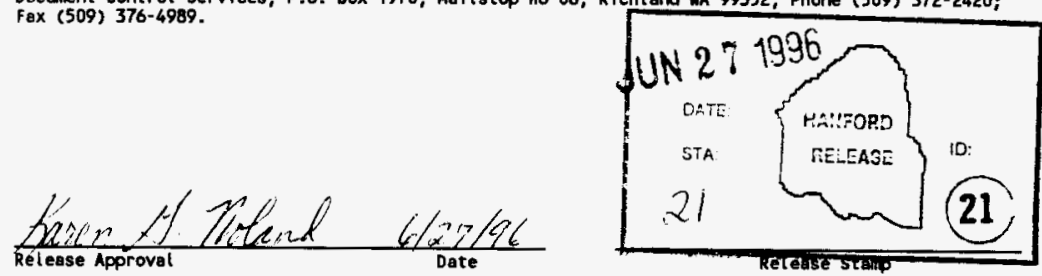

\section{Approved for Public Release}




\title{
UNCERTAINTY ANALYSIS FOR ESTIMATES OF TRAPPED GAS
}

\author{
F. Schmittroth (5-15-96)
}

\section{Executive Summary}

An uncertainty analysis was made for the amount of trapped gas based on the barometric pressure method for the situation where the method indicates small amounts of gas. The derived base uncertainty (expressed as standard deviation) for the amount of trapped gas is found to be where $\sigma_{d L / d P}$ is the standard deviation of $\mathrm{dL} / \mathrm{dP}$ (in./in. Hg) and $w$ is the

$$
\mathbf{\sigma}_{G}=\left(11 \times 10^{3} f t^{3}\right) \times\left(1+\frac{W}{300}\right)^{2} \sigma_{d L / d P}
$$

average waste depth (in.) over the trapped gas. An additional factor was found that includes other sources of uncertainty such as surface geometry effects and overburden pressure:

While $\sigma_{d h d p}$ is normally obtained from the regression analysis used to estimate $\mathrm{dL} / \mathrm{dP}$, the following formula was obtained that illustrates the important sources of uncertainty:

$$
\sigma_{d L / d P}^{2}=\frac{1}{n} \frac{\sigma_{L}^{2}}{\sigma_{p}^{2}},
$$

where $\sigma_{L}^{2}$ is the variance of the surface-level measurements, and $\sigma_{p}{ }^{2}$ is the variance of the pressure fluctuations.

\subsection{Introduction}

Recent work has confirmed that the waste level in Hanford tanks responds to changes in barometric pressure. While it is expected that these changes are a consequence of trapped gas, the quantitative relation has been less clear.

Additional tank-waste characterization is vital to understanding the important phenomena. In the meantime, it is worthwhile to identify as clearly as possible the important parameters and their uncertainties in the context of current tank-waste models. This study addresses these issues and provides semi-quantitative uncertainties for trapped-gas estimates; more specifically for the case where there the waste-level response to barometric pressure changes is small. 


\subsection{Estimation of Trapped Gas}

There are several potential ways to estimate trapped gas: direct measurements as in void measurements, mass balance estimates accounting for production and loss, and other indirect estimates. Two of the latter, based on surface-level measurements, include surface-level rise and correlations with barometric pressure changes.

To maintain a limited scope, this study focuses mainly on the barometric pressure estimates. A brief introduction to mass-balance estimates is given in Appendix $A$.

\subsection{Surface-1evel Mode1}

The tank-waste level, $L_{m}$, is a function of the volume of trapped gas, $V_{g}$, and other possible parameters denoted here by $x$ :

$$
L_{m}=L_{m}\left(V_{g} ; x\right)
$$

The parameters represented by $x$ could include time. A first-order approximation to this general model, is obtained by a Taylor expansion about zero (no trapped gas):

$$
L_{m}=L_{m}(0 ; x)+\frac{\partial L_{m}}{\partial V_{g \circ}} V_{g} .
$$

The absolute amount of gas, G, (expressed as a volume) is related to the gas volume, $V_{g}$, by Boyle's law:

$$
P_{s} G=P_{g} V_{g},
$$

where $P_{s}$ and $P_{g}$ are standard pressure and the in situ gas pressure respectively. "Combining these results gives

where $h_{w 0}=L_{m}(0 ; x)$ reflects the waste level height in the absence of trapped

$$
L_{m}=h_{\text {wo }}+\frac{\partial L_{m}}{\partial V_{g o}}\left(\frac{P_{g}}{P_{g}}\right) G \text {, }
$$

gas.

The simplest waste-level model assumes that the change in waste level gives a volume change equal to the change in gas volume:

$$
A d L_{m}=d V_{g},
$$


where $A$ denotes the area of the tank. The possibility that the waste-level does not quantitatively respond to this volume change can be modeled by introducing an added factor, $\theta$, giving

$$
L_{m}=h_{w o}+\left(\frac{P_{s}}{P_{g}}\right)\left(\frac{\theta}{A}\right) G .
$$

A value $\theta=1$ gives the simplest model. A value $\theta=0$ would correspond to the case where the measured level did not respond to gas volume changes as with a fixed crust. A more complicated example for $\theta$ is given in Appendix $B$ for a waste surface that may change shape. As with $h_{\text {wo }}, \theta$ could be time-dependent.

This first order result represents a moderately general model of the measured waste level that explicitly describes distinct features of the problem. As such, it is a convenient basis to examine sources of uncertainty.

The measured level, $L_{m}$, is expressed as two terms. The first term represents the waste height expected in the absence of trapped gas and includes the geometry of both the waste and measuring devices.

The contribution of trapped gas, $G$, is given by the second term and includes one factor $(\theta / A)$ that accounts for added surface geometry effects of the waste and a second factor $P_{s} / P_{g}$ related to compression of the trapped gas. This factor may be made more transparent by explicitly expressing the gas pressure, $P_{g}$, as the sum of the ambient pressure, $P_{a}$, and an overburden pressure, $P_{b}$ : The overburden pressure can include internal stresses in the waste, surface

$$
P_{g}=P_{a}+P_{b}
$$

tension effects, etc. as well as the direct weight of the waste on the trapped gas.

\subsubsection{Surface-Level Rise Method}

There are two ways to estimate the trapped gas volume, G, from this model: the surface-level rise method and the barometric pressure method. In the surfacelevel rise method, one solves directly for $G$ in terms of the surface height, $L_{m}$, giving:

$$
G=\left(\frac{P_{g}}{P_{s}}\right)\left(\frac{A}{\Theta}\right)\left(L_{m}-h_{w o}\right) .
$$

Since $h_{\text {wo }}$ is not an observable quantity, this equation can only be used to estimate changes in the amount of gas, G. A detailed uncertainty analysis is not carried out here. It is nevertheless clear that the uncertainty in the difference $L_{m}-h_{\text {wo }}$ is a major issue. The value of $G$ (actually $\Delta G$ ) depends on 
WHC-SD-EM-ANAL-001, Rev. 0

Page 4 of 11

changes in the voided waste level, $\Delta h_{\text {wO }}$, as well as changes, $\Delta \mathrm{J}_{m}$, in the measured surface level. The former can include numerous effects such as evaporation and shifts in the waste surface that are difficult to quantify.

\subsubsection{Barometric Pressure Method}

In the barometric-pressure method, one first differentiates with respect to atmospheric pressure. This has a large potential advantage in that the nul1gas waste height, $h_{\text {uo }}$, term is expected to be negligible unless the waste had a compressibility comparable to the trapped gas. The result is With respect to an uncertainty analysis, this expression shares the geometry

$$
G=-\left(\frac{P_{g}^{2}}{P_{s}}\right)\left(\frac{A}{\Theta}\right)\left[1+\frac{d P_{b}}{d P_{a}}\right]^{-1} \frac{d L_{m}}{d P_{a}} .
$$

factor, $(A / \theta)$, and the gas pressure, $P_{g}$, with the surface-level rise equation; al though the square of $P_{g}$ implies a higher sensitivity to $P_{g}$.

For now, the factor $\left[1+\mathrm{dP}_{\mathrm{b}} / \mathrm{dP}_{\mathrm{a}}\right]$ is assumed to be unity as would be the case if the overburden pressure, $P_{b}$, were independent of the atmospheric pressure, $P_{a}$. As an example, for surface tension phenomena one can show that $d P_{b} / d P_{a}$ is on the order of the ratio of induced surface-tension pressure relative to the total gas pressure. The $\left[1+\mathrm{dP}_{\mathrm{b}} / \mathrm{dP}_{\mathrm{a}}\right]$ factor then simply compensates for some of the corresponding increase in the $\mathrm{P}_{\mathrm{g}}^{2}$ factor.

\subsection{Uncertainty Analysis}

\subsection{Standard Deviation, $\sigma_{G}$, for $\&$}

The uncertainty analysis starts from the preceding equation dropping the bracketed factor:

$$
G=-\left(\frac{P_{g}^{2}}{P_{s}}\right)\left(\frac{A}{\theta}\right) \frac{d L_{m}}{d P_{\mathrm{a}}} .
$$

The objective of this analysis is to consider uncertainties for $G$ in the situation where the estimated value for $G$ is near zero. It is tempting to apply the standard textbook recipe for propagating uncertainties of independent variables whereby the total fractional variance is just the sum of the fractional variances for the individual factors. However the conditions for this rule are not met. 
WHC-SD-EM-ANAL-001, Rev. 0

Page 5 of 11

Instead an exact expression for two independent factors can be generalized. The equation for $G$ has the form

$$
f=a^{2} b c
$$

Under suitable assumptions, an expression for the variance of $f$ is derived in Appendix C. Application of this result to the expression for $G$ gives its standard deviation as

$$
\sigma_{G}=k\left(\frac{P_{g}^{2}}{P_{s}}\right)\left(\frac{A}{\theta}\right) \sigma_{d L / d P}
$$

where

$$
\kappa^{2}=1+\frac{\sigma_{1 / \theta}^{2}}{(1 / \theta)^{2}}+4\left(\frac{p_{b}}{P_{g}}\right)^{2} \frac{\sigma_{P_{b}}^{2}}{P_{b}^{2}}+4\left(\frac{p_{b}}{P_{g}}\right)^{2} \frac{\sigma_{1 / \theta}^{2}}{(1 / \theta)^{2}} \frac{\sigma_{P_{b}}^{2}}{P_{b}^{2}}
$$

Assuming that the overburden pressure, $P_{b}$, is about half the total gas pressure, $P_{g}$, gives a more transparent result while retaining the important variable dependencies:

$$
\boldsymbol{\kappa}^{2} \approx 1+\frac{\mathbf{\sigma}_{\boldsymbol{\theta}}^{2}}{\boldsymbol{\theta}^{2}}+\frac{\boldsymbol{\sigma}_{P_{b}}^{2}}{P_{b}^{2}}+\frac{\boldsymbol{\sigma}_{\boldsymbol{\theta}}^{2}}{\theta^{2}} \frac{\boldsymbol{\sigma}_{P_{b}}^{2}}{P_{b}^{2}} .
$$

A second assumption made is that the fractional uncertainty in $1 / \theta$ is approximately given by the fractional uncertainty in $\theta$, an assumption that breaks down when $\theta$ is small.

Simply stated, the base uncertainty, $\sigma_{G}$, of $G$ is given in terms of the uncertainty, $\sigma_{d L / d}$ of $\mathrm{dL} / \mathrm{dP}$ by the same formula used to calculate the amount of gas, $G$, from the value of $d L / d P$. A factor $\kappa$ accounts for other sources of uncertainty (surface geometry effects and overburden pressure). For example, an uncertainty in $\theta$ comparable to an assumed value of $\theta=1$ (no surface geometry correction) would double $\kappa^{2}$ to 2 and increase $\sigma_{G}$ by $\sqrt{2}$.

Note that a substantially more complicated expression would arise if the assumption of a small $\mathrm{dL} / \mathrm{dP}$ were dropped.

\subsection{Standard Deviation, $\sigma_{d L / d P}$, of $d L / d P$}

Given that the uncertainty in $G$ is directly related to the uncertainty in $\mathrm{dL} / \mathrm{dP}$, this section further considers the latter uncertainty. First it should be noted that the $\mathrm{dL} / \mathrm{dP}$ uncertainty is a direct output of regression analysis that determines $\mathrm{dL} / \mathrm{dP}$ from waste-level data vs. barometric pressure data. Nevertheless some insight into the important sources of uncertainty and their 
consequence is gained by a more detailed examination.

In Appendix D, the following expression is derived for the variance of $\mathrm{dL} / \mathrm{dP}$ :

$$
\sigma_{d L / d P}^{2}=\frac{1}{n} \frac{\sigma_{L}^{2}}{\sigma_{p}^{2}},
$$

where $\sigma_{L}$ is the standard deviation of the level measurement uncertainties, and $\sigma_{p}$ is the standard deviation of the pressure fluctuations. The number of data points in the least-squares fit is $n$. This expression clearly shows that when the barometric pressure is stable (small $\sigma_{\mathrm{p}}$ ), the uncertainty in $\mathrm{dL} / \mathrm{dP}$ becomes large.

To show this effect more quantitatively, the fluctuations in hourly barometric pressure measurements were computed over the last 15 years. The computed average was $29.2 \mathrm{in.Hg}$ with a standard deviation of $0.3 \mathrm{in}$. Hg. However typical pressure fluctuations are much smaller during shorter time intervals. Roughly half the time intervals have fluctuations less than $0.1 \mathrm{in.Hg}$ if values are computer for 100 hour time intervals. On the other hand, during the passage of a strong cold front, pressure changes of $1 \mathrm{in.Hg}$ are possible.

Using the relatively low value of $\sigma_{\mathrm{p}}=0.1$ in. Hg that might be expected if random measurements were made, the following table of $\mathrm{dL} / \mathrm{dP}$ values was constructed.

Table 1. Std. Dev. for $\mathrm{dL} / \mathrm{dP}\left(\sigma_{\mathrm{p}}=0.1\right.$ in. $\left.\mathrm{Hg}\right)$

\begin{tabular}{|l|l|l|l|}
\hline & & \multicolumn{2}{|c|}{$\sigma_{\text {dlddip }}$} \\
\hline & $\sigma_{1}$, in. & $n=10$ & $n=100$ \\
\hline ENRAF & 0.01 & 0.03 & 0.01 \\
\hline FIC & 0.10 & 0.32 & 0.10 \\
\hline Man. Tape & 0.25 & 0.79 & 0.25 \\
\hline
\end{tabular}

Typical values of $\mathrm{dL} / \mathrm{dP}$ when there is a significant barometric indication of trapped gas are in the range of a few tenths (ir. waste)/(in. Hg). The uncertainties in the table thus show that, for routine pressure variations, the manual tape measurements are unlikely to detect trapped gas while the FIC uncertainties are consistent with detection. The ENRAF values should be very good if the quoted measurement uncertainties are valid in practice. These results are consistent with observed practice and add additional support for the barometric pressure methodology. 


\subsection{Results}

A simple example illustrates the use of the above results. As discussed, uncertainties in the amount of trapped gas are calculated from uncertainties in $\mathrm{dL} / \mathrm{dp}$ using the same formula used to calculate the of the amount of trapped gas from $\mathrm{dL} / \mathrm{dP}$. If other uncertainties are ignored, the formula reduces to: Recall this result assumes $(k=1)$ that there are no uncertainties in the

$$
\boldsymbol{\sigma}_{G}=\left(\frac{P_{g}^{2}}{P_{s}}\right)\left(\frac{A}{\boldsymbol{\theta}}\right) \sigma_{d L / d P} .
$$

surface-1evel geometry factor, $\theta$, or the overburden pressure, $P_{b}$. Other assumptions are that the compressibility of the voided gas is small and that the overburden pressure is independent of the ambient pressure.

This result can be further simplified by noting that typical waste densities are close to $1 / 10$ the density of mercury $\left(p_{\mathrm{Hg}}=13.6 \mathrm{~g} / \mathrm{cm}^{3}\right)$. Then to a good approximation and assuming a standard pressure rounded to $P_{s}=30$ in. $\mathrm{Hg}$, one has where $w$, given in inches, is the average depth of the waste over the trapped

$$
\frac{P_{g}}{P_{s}}=\left(1+\frac{P_{b}}{P_{s}}\right)=\left(1+\frac{w / 10}{30}\right) \text {, }
$$

gas.

Consider a $75 \mathrm{ft}(22.9 \mathrm{~m})$ diameter tank. Assuming the surface level responds as a piston to volume changes in trapped gas $(\theta=1)$ one readily obtains

$$
\sigma_{G}=\left(11 \times 10^{3} f t^{3}\right) \times\left(1+\frac{W}{300}\right)^{2} \sigma_{d L / d P}
$$

where $o_{d L / d P}$ is given in units of (in./in. $\mathrm{Hg}$ ) and $w$ is given in inches.

Thus, for example, a barometric-pressure analysis indicating little trapped gas but having a dL/dP uncertainty of 0.1 in./in. $\mathrm{Hg}$ (see Table 1) would give a standard deviation of about

$$
\sigma_{G}=4400 f t^{3}
$$

for a waste depth of $w=300$ inches. 
A graphical depiction of $\sigma_{G}$ as a function of the average depth of trapped gas is shown in the figure below. Curves are given for ENRAF, FIC, and manual tape measurements corresponding to $\sigma_{\mathrm{dL} / \mathrm{dp}}$ values of $0.01,0.1$, and 0.25 respectively (see Table I.).

Figure 1. Uncertainty in Trapped Gas Estimates for Measured Values Near Zero.

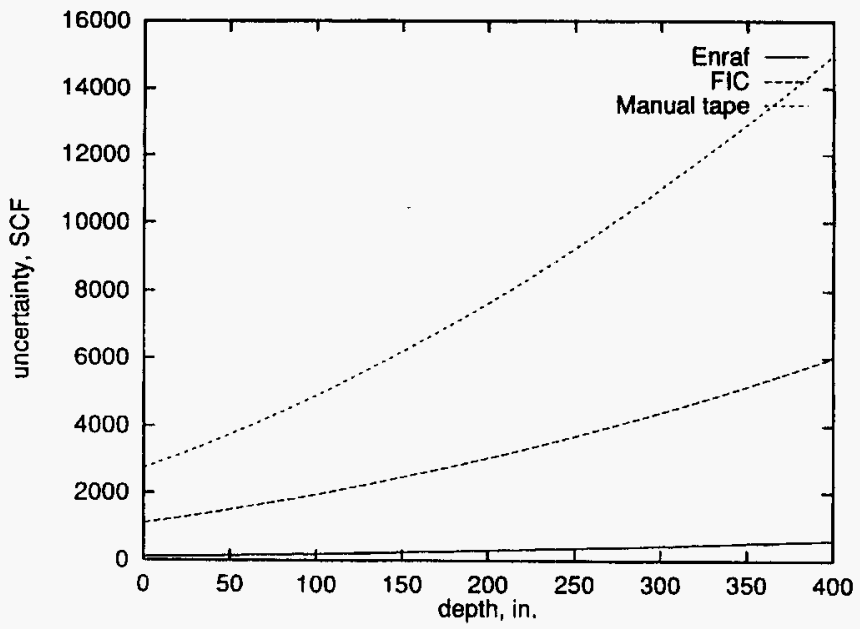




\section{Appendix A: Mass-balance Equations}

The amount of trapped gas, $G(t)$, is equal to an initial amount $G_{0}$ corrected by production and release term:

$$
G(t)=G_{0}+\int_{0}^{t} p\left(t^{\prime}\right) d t^{\prime}-\int_{0}^{t} g\left(t^{\prime}\right) d t^{\prime},
$$

where $p(t)$ is a gas production rate. The gas release rate, $g(t)$, can be expressed in terms of fractional gas release model, $r(t)$ by

$$
g(t)=r(t) G(t) \text {. }
$$

A simple production model would be constant production: $p(t)=$ constant. Two simple release models are 1) constant release, $\alpha$ :

$$
I(t)=\alpha \text {, }
$$

and 2) episodic release:

$$
r(t)=\sum_{i} \alpha_{i} \delta\left(t-t_{i}\right)
$$

where $\alpha_{i}$ is a fractional release at time $t_{i}$. These models can be used to estimated the amount and release rates of trapped gas.

\section{Gas concentration}

Given the gas release rate, $g(t)$, and a fresh air mixing rate, $f(t)$, the volume of dome gas, $V_{G}$, can then be obtained by equating the time-rate-ofchange of $V_{G}$ to the dome production and loss terms:

$$
\frac{d V_{G}}{d t}=g(t)-\left(\frac{V_{G}}{V}\right) e
$$

where

$$
\begin{aligned}
& V=V_{G}+V_{f} \\
& e=g+E .
\end{aligned}
$$

These equations are included for completeness and their solution is not pursued. 


\section{Appendix B: A Surface-1evel Model}

A lensing of the waste surface is used to illustrate a simple surface geometry model. Let the surface of the waste be defined as the sector of a sphere of radius $r$. Let the height of the sector be $h$. Then the sector volume is given by

$$
V=\frac{1}{3} \pi h^{2}(3 r-h)
$$

Further assume that level gauge contacts the lens-shaped surface of the waste at a distance $x$ from the center of the tank. A waste-height correction factor at the gauge location is then found by equating the sector volume to the volume of a piston. After some geometry calculations and using a first-order approximation, A height correction is found:

$$
\theta=2\left(1-\frac{x^{2}}{R^{2}}\right)
$$

where $R$ is the tank radius.

For example, in this model the lensed height would be twice the height of an equivalent piston at the center of the tank $(x=0)$ decreasing to zero at the tank edge $(x=R)$.

Appendix $c$ : Variance of $a^{2} b c$

Let

$$
f=a^{2} b c
$$

The variance of this expression can be obtained by repeated application of the two factor formula for variance:

$$
\sigma_{g}^{2}=a^{2} \sigma_{b}^{2}+b^{2} \sigma_{a}^{2}+\sigma_{a}^{2} \sigma_{b}^{2}
$$

where

$$
g=a b
$$

This expression is distribution free and assumes only that correlations between the factors may be neglected.

The resulting expression for the variance, $\sigma_{f}{ }^{2}$, of $f$ is somewhat complicated and can be substantially simplified by focusing on the specific case where the 
estimated value for $c$ is small compared to its standard deviation $\left(c<\sigma_{c}\right)$. The results is

$$
\sigma_{f}^{2} \approx a^{4} b^{2} \sigma_{c}^{2} K^{2}
$$

where $\sigma_{c}^{2}$ denotes the variance of $c$, and

$$
\kappa^{2}=1+\frac{\sigma_{b}^{2}}{b^{2}}+4 \frac{\sigma_{a}^{2}}{a^{2}}+4 \frac{\sigma_{a}^{2}}{a^{2}} \frac{\sigma_{b}^{2}}{b^{2}}
$$

includes the variances, $\sigma_{a}^{2}$ and $\sigma_{b}^{2}$ of $a$ and $b$ respectively. Here $a$ and $b$ denote mean values. The factor of four occurs because "a" appears as a squared value in the expression for $f$.

\section{Appendix D: Standard Deviation, $\sigma_{\mathrm{dL} / \mathrm{dP}}$, of $\mathrm{dL} / \mathrm{dP}$}

Recast the linear-regression model in the form

$$
L_{i}=L_{0}+\left(P_{i}-P_{0}\right) \frac{d L}{d P}+\epsilon_{I}
$$

where $\left\{L_{i}, P_{i}\right\}$ are a set of measured waste-level barometric pressure couples at $n$ points and $\epsilon_{\mathrm{L}}$ represents random errors in the measured levels.

Standard regression texts give the following result for the variance in the fitted $\mathrm{dL} / \mathrm{dP}$ value:

$$
\sigma_{d L / d P}^{2}=\sigma_{L}^{2} \frac{n}{n \sum_{i} P_{i}^{2}-\left(\sum_{i} P_{i}\right)^{2}}
$$

where $\sigma_{\mathrm{L}}^{2}$ is the variance in the level measurements. The second factor, however, is just $1 / n \sigma_{p}{ }^{2}$ where $\sigma_{p}{ }^{2}$ is the variance of the atmospheric pressure fluctuations. Thus one finds

$$
\mathbf{\sigma}_{d L / d P}^{2}=\frac{1}{n} \frac{\mathbf{\sigma}_{L}^{2}}{\mathbf{\sigma}_{P}^{2}} .
$$

As one expects, small measurement uncertainties and large pressure variations both reduce the uncertainty in $\mathrm{dL} / \mathrm{dP}$. 


\begin{tabular}{|c|c|c|c|c|c|}
\hline \multicolumn{6}{|c|}{ DISTRIBUTION SHEET } \\
\hline \multirow{2}{*}{$\begin{array}{l}\text { To } \\
\text { Distribution }\end{array}$} & \multirow{2}{*}{\multicolumn{3}{|c|}{$\begin{array}{l}\text { From } \\
\text { Criticality and Shielding }\end{array}$}} & \multicolumn{2}{|l|}{ Page 1 of 1} \\
\hline & & & & \multicolumn{2}{|l|}{ Date } \\
\hline \multirow{2}{*}{\multicolumn{4}{|c|}{$\begin{array}{l}\text { Project Title/Work Order } \\
\text { Uncertainty Analysis for Estimates of Trapped Gas }\end{array}$}} & \multicolumn{2}{|c|}{ EDT No. $\quad 616560$} \\
\hline & & & & \multicolumn{2}{|l|}{ ECN No. } \\
\hline \multicolumn{2}{|l|}{ Name } & $\begin{array}{l}\text { Text } \\
\text { With All } \\
\text { Attach. }\end{array}$ & Text Only & $\begin{array}{l}\text { Attach./ } \\
\text { Appendix } \\
\text { Only }\end{array}$ & $\begin{array}{l}\text { EDT/ECN } \\
\text { Only }\end{array}$ \\
\hline W. B. Barton & R2-11 & $x$ & & & \\
\hline J. Greenborg & HO-35 & $x$ & & & \\
\hline J. D. Hopkins & R2-11 & $x$ & & & \\
\hline D. M. Ogden & HO-34 & $x$ & & & \\
\hline A. L. Ramble & A3-38 & $x$ & & & \\
\hline F. A. Schmittroth & HO-35 & $x$ & & & \\
\hline P. D. Whitney & $\mathrm{K} 5-12$ & $x$ & & & \\
\hline Central Files (Original +1 ) & A3-88 & $x$ & & & \\
\hline
\end{tabular}

